Journal of

Synchrotron

Radiation

ISSN 0909-0495

Received 8 December 2004

Accepted 19 April 2005

C) 2005 International Union of Crystallography Printed in Great Britain - all rights reserved

\section{$X$-ray diffraction method for the investigation of contacts between solid surfaces}

\author{
H. R. Keymeulen,* T. Lackner, A. Diaz, B. D. Patterson and J. F. van der Veen \\ Paul Scherrer Institut, CH-5232 Villigen PSI, Switzerland. E-mail: heilke.keymeulen@psi.ch
}

\begin{abstract}
A coherent X-ray scattering method for investigating the formation of the contact region between two solid surfaces is presented. Diffraction of X-rays from two crossed cylindrical quartz surfaces, coated with $\mathrm{Cr}$ and $\mathrm{TiO}_{2}$, revealed a total contact area of $90 \pm 10 \mu \mathrm{m}$. In the so-called Hertz model for two surfaces in non-adhesive contact, this value is directly related to the displacement of the surfaces and the applied external force. Values of $40 \pm 3 \mathrm{~nm}$ for the displacement and $24 \pm 3 \mathrm{mN}$ for the force are found. The method is also useful for studying liquids in confinement.
\end{abstract}

Keywords: X-ray diffraction; Hertzian contact; confined fluids.

\section{Introduction}

During the last half-century a true revolution has occurred in the science of friction, lubrication and wear, commonly known as tribology. The interplay between the development of new experimental techniques and new theoretical insights stimulated a shift to a new field, called nanotribology, that aims at understanding friction between two solid surfaces on the molecular scale, both in the presence and absence of fluids. For an overview of the experimental and theoretical progress made in this field we refer to Müser et al. (2003). One very well known tool is the surface force apparatus (SFA), that was developed to study contact regions between surfaces. The sizes of the contacts under investigation with this apparatus can range from 10 to $100 \mu \mathrm{m}$. In the SFA the distance between two curved molecularly smooth mica surfaces can be controlled to sub-angstrom precision. The surfaces have a cylindrical shape and are mounted with the axes crossed. The crossed cylinders form a disc-like contact region. By use of a set of springs, shear and normal forces between the surfaces can be measured. Also, fluids in confinement between the mica surfaces have been investigated with the SFA. The number of experimental studies on confined fluids with the SFA is growing steadily (Heuberger et al., 2001; Qiao \& Christenson, 1999, 2001; Becker \& Mugele, 2003; Lin \& Granick, 2003; Spikes \& Granick, 2003).

Here we report observations of X-ray diffraction from the contact region of two crossed cylindrical surfaces. This experiment is in the first place a reference for future X-ray diffraction studies of confined molecular liquids, and in the second place can be considered a method of measuring the degree of coherency of the X-ray beam. Coherent X-ray diffraction effects from many objects have been studied, from pinholes and slits (Leitenberger et al., 2003, 2004; Panzner et al., 2003; Livet et al., 2003) or from fibres (Kohn et al., 2000).

\section{Experimental}

The crossed-cylinder geometry is locally equivalent to a sphere near a flat surface. Upon touching, a disc-like contact zone is formed.

Quartz $\left(\mathrm{SiO}_{2}\right)$ cylinders of radius $10.3 \mathrm{~mm}$ were coated with a layer of $\mathrm{Cr}$ and a layer of $\mathrm{TiO}_{2}$ of thicknesses $25 \mathrm{~nm}$ and $500 \mathrm{~nm}$, respectively. The layers serve for optical interferometry. $\mathrm{TiO}_{2}$ has a high absorption coefficient for X-rays, which helps to observe diffraction effects. Multiple-beam interferometry is a standard technique for measuring the distance between the surfaces in an absolute way (Tolansky, 1970).

The quartz surfaces were bought from Mark Optics. The surface roughness was smaller than $10 \AA$ A. First, the quartz surfaces were cleaned extensively using acetone. Then they were brought into a sputtering device where they were first plasma-cleaned for $5 \mathrm{~min}$ in an Ar plasma, and then the $\mathrm{Cr}$ and $\mathrm{TiO}_{2}$ layers were sputtered at low Ar pressure $\left(10^{-4} \mathrm{~Pa}\right)$. The r.m.s. roughness and the figure of error of the surfaces were measured using an interference microscope and were $3 \AA$ and $0.001^{\circ}$, respectively. Actually, this is the roughness and the figure of error for the $\mathrm{Cr}$ layer, since $\mathrm{TiO}_{2}$ is transparent in the optical spectrum. The surfaces were mounted in the SFA, which was then transported to the beamline. Fig. 1 shows the experimental set-up for the diffraction experiment, which was carried out at the materials science wiggler beamline at the Swiss Light Source (Patterson et al., 2005). The X-ray energy was $12.4 \mathrm{keV}$. The flux at this energy is $9.0 \times 10^{9}$ photons s$^{-1}$ $\mathrm{mm}^{-2}$ for a non-focused beam (spot size $9 \mathrm{~mm} \times 100 \mathrm{~mm}$ ). The source divergence is $0.23 \mathrm{mrad}$ vertically and $2.5 \mathrm{mrad}$ horizontally. The SFA was mounted on a five-axis surface diffractometer (Newport). The surfaces of the SFA were mounted such that the axis of the lower cylinder was parallel to the X-ray beam. Aligning the cylinders in the X-ray beam 


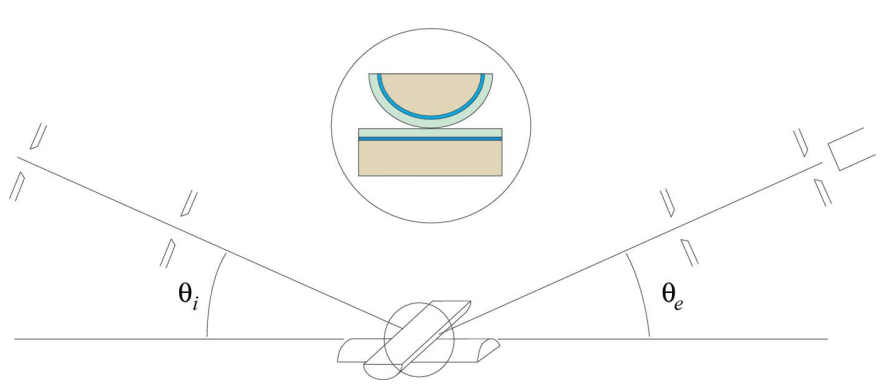

Figure 1

Alignment of the crossed cylinders in the X-ray beam. Slits define the beam size. The incoming angle $\theta_{i}$ was set to $0.12^{\circ}$. On the detector we see a range of outgoing angles $\theta_{e}$. The inset shows the crossed cylinders coated with $\mathrm{Cr}$ and $\mathrm{TiO}_{2}$.

was not trivial, but by making use of the reflection from the lower surface it could be performed to within $5 \mathrm{mdeg}$. The upper surface was mounted on a double leaf spring and could be positioned vertically using motors. After the alignment the upper surface was brought into contact with the lower one (the leaf springs prevent damage to the $\mathrm{TiO}_{2}$ layer). The CCD camera, a 2048 pixel $\times 2048$ pixel Hystar (magnifying taper 1:3, effective pixel size $4.36 \mu \mathrm{m}$ ), was mounted on the detector arm at $1200 \mathrm{~mm}$ from the sample, implying a subtended angle per pixel of $3.63 \mu \mathrm{rad}$.

In front of the sample, at $20 \mathrm{~cm}$, two pairs of slits were mounted (horizontal and vertical). After the sample, in front of the detector, another pair of slits was available. The sagittal and meridional focusing optics of the beamline were switched off in order to produce the minimum vertical divergence. For this energy the refractive index $n$ of $\mathrm{TiO}_{2}$ is $1-\delta-\beta=$ $1-5.538 \times 10^{-6}-i \times 1.283 \times 10^{-7}$, the absorption length $l_{\mathrm{abs}}=\lambda /(4 \pi \beta)=62 \mu \mathrm{m}$ and the critical angle for total reflection $\alpha_{\mathrm{c}}=(2 \delta)^{1 / 2}=0.19^{\circ}$. The sample was tilted by an angle $\alpha=0.12^{\circ}$, still below $\alpha_{\mathrm{c}}$. A clear reflection and part of the direct beam were seen on the CCD camera. Where the two surfaces were touching, the direct beam was obscured and the reflection had a dark region. The pre-sample slits were set to select a horizontal region of interest that was $500 \mu \mathrm{m}$ wide. Both the reflected and direct beams were then obscured by the detector slits, and an exposure time of $10 \mathrm{~min}$ was necessary to obtain the Fraunhofer diffraction pattern shown in Fig. 2(a). We also attempted to observe diffraction at an incoming angle beyond the critical angle $\left(\alpha=0.4^{\circ}\right)$, but no features were seen. Owing to the longer path lengths through the multilayer, most of the X-rays are absorbed.

\section{Results}

Fig. 2(a) shows the measured diffraction pattern from the crossed cylinders in contact. The well resolved fringes in the side lobes have a spacing which is inversely proportional to the local gap width. As the gap widens at either side of the touching point between the cylinders, the fringe spacing becomes smaller. The central part of the pattern shows a broad plateau, which we attribute to the formation of a pressure contact, effectively widening the contact region. A fringe pattern is also seen in the plateau, implying interference of the X-rays travelling through the contact region. One could speak of a 'soft slit', reflecting the height-dependent absorption at the curved edge of the upper cylinder. There is also a second class of broader horizontal fringes in the side lobes, with the same periodicity as the fringes in the central part. It is unclear how these arise, but the similarity with the central part suggests also a 'soft-edge' effect at the upper cylinder.

Fig. 2(b) shows a fit to the measured data. For the calculation of the fringe pattern in the side lobes, we propagate the $\mathrm{X}$-rays through a two-dimensional spherical-flat geometry (see inset in Fig. 1). Further details about the propagation are described in the following section. Fringe patterns for a series of gap sizes in the range from 0 to $1.2 \mu \mathrm{m}$ were obtained. The refractive index on both sides of the gap was that of $\mathrm{TiO}_{2}$. After reflection on the lower surface, the parallel beam will become fan-like. This effect is seen on the detector as a broadening of the diffraction pattern in the horizontal plane. To first-order approximation, the horizontal position on the detector at which a reflected ray arrives is given by the formula $y+L \theta_{i} y / R$, where $L$ is the distance from the sample to the detector, $\theta_{i}$ is the incoming angle, $y$ is the distance from the

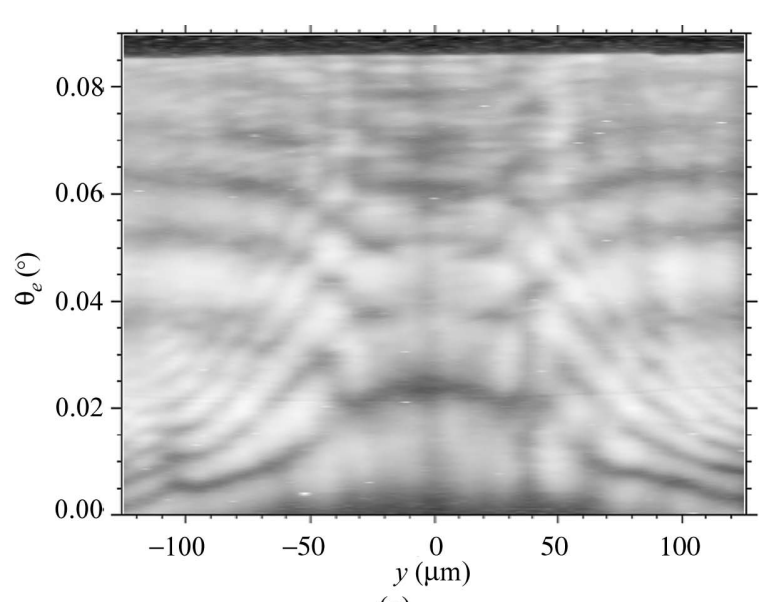

(a)

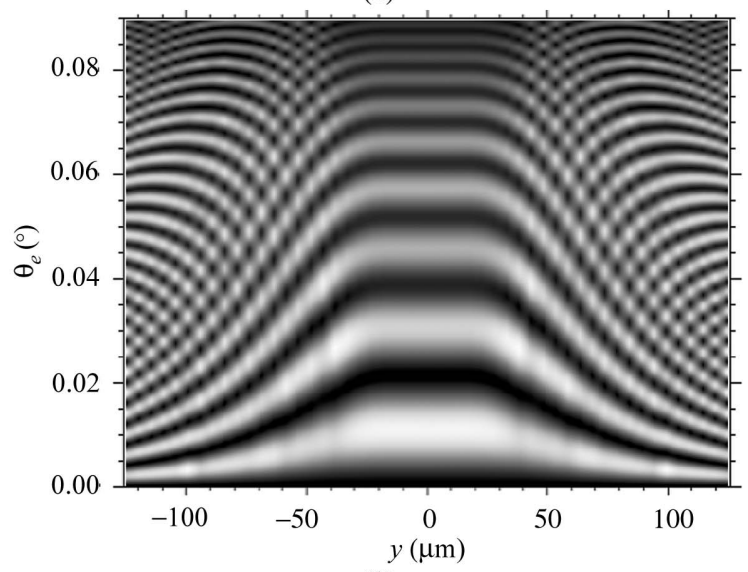

Figure 2

(b)

Diffraction pattern from a crossed-cylinder configuration: (a) experimental pattern and $(b)$ calculated pattern using the wave-propagation formula described in the text. $y$ is the horizontal distance from the center of the contact area, calculated from the corresponding detector position with the formula mentioned in the text. 
center of the contact area, at which the incoming ray is reflected, and $R$ is the radius of curvature of the cylinder. The abscissas of Figs. 2(a) and 2(b) refer to $y$, which is calculated from the horizontal position on the detector using the abovementioned formula. To describe the form of the contact region we assumed the validity of the Hertz model (see below). We assumed a rotationally symmetric contact region in the horizontal plane, and we found the best agreement for a diameter of $90 \pm 10 \mu \mathrm{m}$, which was verified interferometrically using visible light. The fringes in the side lobes are well reproduced by the calculation, although, as noted above, the broader horizontal fringes could not be explained. Instead, a sort of 'secondary' fringe pattern, with much smaller periodicity, appears as the gap size becomes larger. These secondary fringes are not seen in the measurement, perhaps owing to limited angular resolution. In Fig. 3 we show the projected interference pattern for the central data. This contains all the information about the formation of the contact region. For a discussion we refer to the final section.

We also mentioned that this method is useful for estimating the coherence length of the X-ray beam. In the measured fringe pattern in Fig. 2(a) a gap of width up to $1.2 \mu \mathrm{m}$ is resolved, which means that the vertical spatial coherence length $\xi_{\mathrm{v}}$ of the X-ray wavefront is at least this size. Of course, this is not a very useful estimation when we know that the vertical transversal coherence length $\xi_{\mathrm{v}}$ should be in the range $25-100 \mu \mathrm{m}$. To have a better angular resolution, the distance from the sample to the detector must be increased. A sampleto-detector distance of $5000 \mathrm{~mm}$ and a pixel size of $4.36 \mu \mathrm{m}$ correspond to an angular resolution $\Delta \theta$ of $0.87 \mu \mathrm{rad}$, giving a spatial resolution of $\lambda / \Delta \theta \simeq 115 \mu \mathrm{m}$, which is enough to measure the coherence length accurately. We also want to point out that it is possible to measure the horizontal coherence length by mounting the SFA sideways, instead of upright like we did here. A typical value for the horizontal coherence length $\xi_{\mathrm{h}}$ at this beamline is $3-10 \mu \mathrm{m}$.

\section{Wave propagation}

There are several approaches for calculating the wave propagation through a thick phase object. Widely used, for example, is the numerical solution of the two-dimensional Helmholtz equation for objects with a general refractive index profile $n$ in the plane of propagation. We apply wave optics and some appropriate approximations to obtain an analytical expression for the Fraunhofer diffraction pattern. The diffracted amplitude in the far field is

$$
\begin{aligned}
\psi\left(\theta_{i}, \theta_{e}\right)= & C \int_{-\delta}^{\infty} \mathrm{d} x \exp \left[i \varphi\left(x, \theta_{i}, \theta_{e}\right)\right] \exp \left[i k\left(\theta_{i}+\theta_{e}\right) x\right] \\
& -\exp \left[i \varphi\left(x, \theta_{i},-\theta_{e}\right)\right] \exp \left[i k\left(\theta_{i}-\theta_{e}\right) x\right] \\
& -\exp \left[i \varphi\left(x,-\theta_{i}, \theta_{e}\right)\right] \exp \left[i k\left(-\theta_{i}+\theta_{e}\right) x\right] \\
& +\exp \left[i \varphi\left(x,-\theta_{i},-\theta_{e}\right)\right] \exp \left[-i k\left(\theta_{i}+\theta_{e}\right) x\right] .
\end{aligned}
$$

This is the Helmholtz-Kirchoff integral for Fraunhofer diffraction. The phase and amplitude of the incoming wavefront are distorted by the object, here the crossed cylinders, and the Fourier transform of the distorted field gives the farfield diffraction pattern. Looking at Fig. 4, it becomes clear that the four terms take into account the pre- and postreflections at the lower surface. We consider the case of total reflection at the lower cylinder. The phase $\varphi\left(x, \theta_{i}, \theta_{e}\right)$ depends on the optical path length through the upper surface. From the symmetry of the crossed-cylinder geometry it is clear that inverting the path of the rays must result in a mirror plane (in Fig. 4 the plane $A$, parallel to the $X Y$ plane). The optical path length $l+l^{\prime}$ (see Fig. 4) can be derived using simple geometry (we neglect refraction),

$$
\begin{aligned}
l & =t p+p q+q r, \\
t p & =\left[R^{2}-\left(R-x^{\prime}\right)^{2} \cos ^{2} \theta_{i}\right]^{1 / 2}, \\
p q & =\left(R-x^{\prime}\right) \sin \theta_{i}, \\
q r & =b / \cos \theta_{i}, \\
x^{\prime}=q v & =x+\delta+b \tan \theta_{i} .
\end{aligned}
$$

$l^{\prime}$ can be derived analogously: $\theta_{i}$ has only to be replaced by $\theta_{e}$. To first-order approximation, $\varphi\left(x, \theta_{i}, \theta_{e}\right)$ then becomes

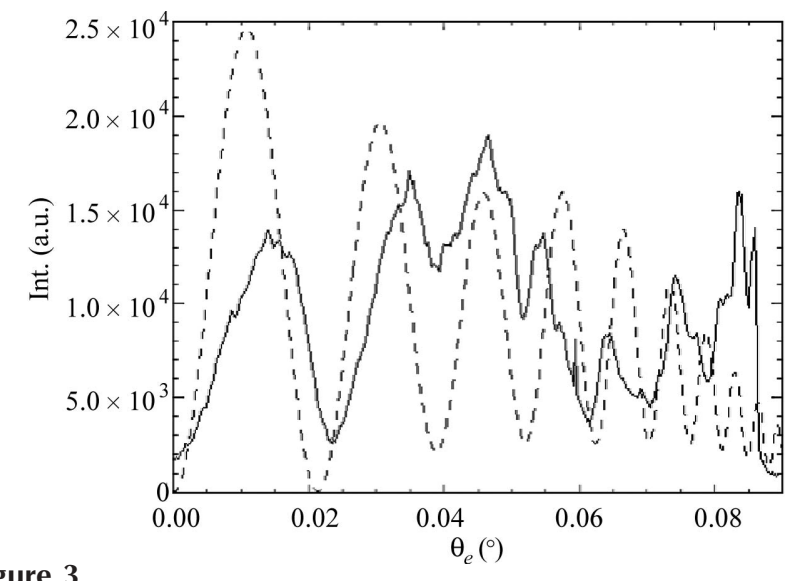

Figure 3

The average of the central part of Fig. 2: experimental data (solid line); fit for a Hertzian contact with a diameter of $90 \mu \mathrm{m}$ (dashed line).

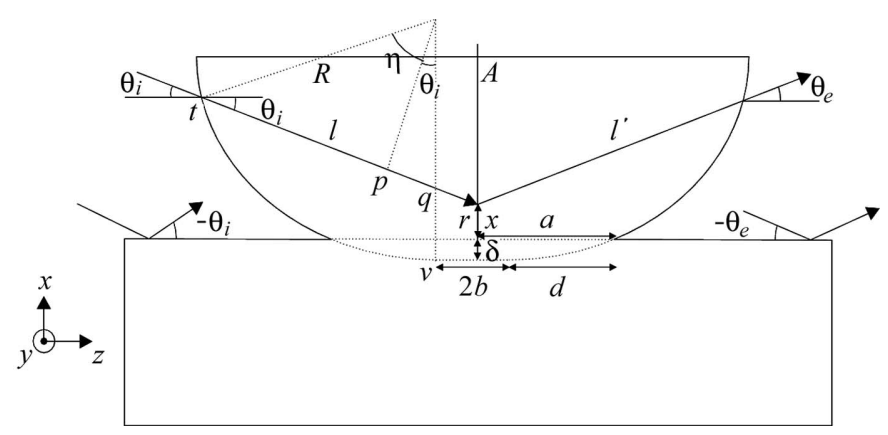

Figure 4

Model for deriving the mathematical expression for the phase $\varphi$, equation (3). The Hertz model describes the contact region for a displacement $\delta$ and a contact radius $d$. A supplementary parameter $b$ was included to explain the central plateau in the diffraction pattern of Fig. 2(a). 


$$
\begin{aligned}
\varphi\left(x, \theta_{i}, \theta_{e}\right)= & k \Delta n\left(l+l^{\prime}\right) \\
= & k \Delta n\left\{\left[2 R\left(x+\delta+b \theta_{i}\right)+R^{2} \theta_{i}^{2}\right]^{1 / 2}+R \theta_{i}+b\right. \\
& \left.+\left[2 R\left(x+\delta+b \theta_{e}\right)+R^{2} \theta_{e}^{2}\right]^{1 / 2}+R \theta_{e}+b\right\},
\end{aligned}
$$

where $\Delta n$ is the difference in the refractive index between air and $\mathrm{TiO}_{2}$ and $k=2 \pi / \lambda$. The angles $\theta_{i}$ and $\theta_{e}$ are the incoming and outgoing angles, respectively, $b$ is the radius of the plateau in the central part, $x$ is the vertical coordinate, originating at the lower surface, $\delta$ is the displacement of the surfaces and $R$ is the radius of the upper surface. A remark on the validity of equation (3) should be made: neglecting refraction is only valid when $\eta \gg \theta_{c}$. As $\sin \eta=t p / R$, this leads, to first-order approximation, to the condition $x^{\prime} \gg\left(\theta_{c}^{2}-\theta_{i}^{2}\right) R / 2$. For an incoming angle $\theta_{i}=0.12^{\circ}$ and a critical angle $\theta_{c}=0.19^{\circ}$ for $\mathrm{TiO}_{2}$, we find $x^{\prime} \gg 34 \mathrm{~nm}$. Therefore in our approximation we do not take into account the refraction from the first $34 \mathrm{~nm}$ of the upper surface.

\section{Discussion and conclusion}

The central part of Fig. 2(a) contains information on the formation of the contact region. For the fitting in Fig. 3, we have already mentioned the Hertz model. This is the simplest model for an elastically deformable sphere on a rigid surface (see Fig. 4). For a treatment of the Hertz theory, see Israelachvili (1991). The following equations for the contact radius and the central displacement can be derived,

$$
\begin{aligned}
\text { contact radius : } & d^{3}=R F / K, \\
\text { displacement : } & \delta=F / K d .
\end{aligned}
$$

$R$ is the radius of the sphere, $K$ is the elastic modulus of the sphere and the surface and $F$ is the external load. Searching for the best resemblance with the experimental data, we find that a plateau of width $2 b=32 \pm 6 \mu \mathrm{m}$, where the upper surface is flattened, should be taken into account in the simulation. Further, for a displacement $\delta$ of $40 \pm 3 \mathrm{~nm}$ the fit in Fig. 3 was obtained. Using (4) and (5) we find a contact radius $d$ of $29 \pm 2 \mu \mathrm{m}$ and a total contact area $(2 a=2 d+2 b)$ of $90 \pm$ $10 \mu \mathrm{m}$. For the case $R=10.3 \mathrm{~mm}$ and $K=10.4 \mathrm{GPa}$ for $\mathrm{TiO}_{2}$, the Hertz theory gives an external force of $24 \pm 3 \mathrm{mN}$ [equation (5)]. The deviations give an upper and lower boundary value for which acceptable agreement is obtained.

We conclude that the Hertz model confirms the observed value for the contact diameter of approximately $90 \mu \mathrm{m}$.
However, this parameter can more easily be determined using microscopy. More interesting is that the displacement parameter $\delta$ is a direct fit parameter. Fig. 3 shows how X-rays penetrate the contact region. We attribute the differences between theory and experiment to the figure of error of the cylinder surfaces, which was not taken into account in our model. Despite this shortcoming, we believe this scattering technique is sensitive enough to make reasonable estimates of the geometry of any kind of contact between two surfaces. Extensions to more complicated models that take into account adhesion are possible. Instead of an analytical expression for the diffraction, an advanced numerical approach would then be more favorable as a model for the wave propagation.

The authors would like to acknowledge M. Horisberger for sputtering the quartz surfaces.

\section{References}

Becker, T. \& Mugele, F. (2003). Phys. Rev. Lett. 91, 166104-1-1661044.

Heuberger, M., Zach, M. \& Spencer, N. D. (2001). Science, 292, 905908.

Israelachvili, J. N. (1991). Intermolecular and Surface Forces. New York: Academic Press.

Kohn, V., Snigireva, I. \& Snigirev, A. (2000). Phys. Rev. Lett. 85, 2745 2748.

Leitenberger, W., Wendrock, H., Bischoff, L., Panzner, T., Pietsch, U., Grenzer, J. \& Pucher, A. (2003). Physica B, 336, 63-67.

Leitenberger, W., Wendrock, H., Bischoff, L. \& Weitkamp, T. (2004). J. Synchrotron Rad. 11, 190-197.

Lin, Z. \& Granick, S. (2003). Langmuir, 19, 7061-7070.

Livet, F., Bley, F., Ehrburger-Dolle, F., Geissler, E., Lebolloc, D. \& Schulli, T. (2003). J. Appl. Cryst. 36, 774-777.

Müser, M. H., Urbakh, M. \& Robbins, M. O. (2003). Advances in Chemical Physics, Vol. 126, edited by I. Prigogine and S. A. Rice, pp. 187-272. New York: Wiley.

Panzner, T., Leitenberger, W., Grenzer, J., Bodenthin, Y., Geue, Th., Pietsch, U. \& Möhwald, H. (2003). J. Phys. D, 36, A93-A97.

Patterson, B. D., Abela, R., Auderset, H., Chen, Q., Fauth, F., Gozzo, F., Ingold, G., Kühne, H., Lange, M., Maden, D., Meister, D., Pattison, P., Schmidt, Th., Schmitt, B., Schulze-Briese, C., Shi, M., Stampanoni, M. \& Willmott, P. R. (2005). Nucl. Instrum. Methods Phys. Res. A, 540, 42-67.

Qiao, Y. \& Christenson, H. K. (1999). Phys. Rev. Lett. 83, 1371-1374.

Qiao, Y. \& Christenson, H. K. (2001). Phys. Rev. Lett. 86, 3807-3810.

Spikes, H. \& Granick, S. (2003). Langmuir, 19, 5065-5071.

Tolansky, S. (1970). Multiple Beam Interferometry of Surfaces and Films. New York: Dover. 